\title{
Laser detection utilizing coherence
}

David M. Benton, Marie A. Zandi, Kate Sugden

David M. Benton, Marie A. Zandi, Kate Sugden, "Laser detection utilizing coherence," Proc. SPIE 11161, Technologies for Optical Countermeasures XVI, 111610G (7 October 2019); doi: 10.1117/12.2532058

SPIE. Event: SPIE Security + Defence, 2019, Strasbourg, France 


\title{
Laser Detection Utilizing Coherence
}

\author{
David M. Benton ${ }^{1}$, Marie A. Zandi and Kate Sugden \\ Aston Institute of Photonic Technologies, Aston University, Aston Triangle, Birmingham, B4 7ET, UK \\ ${ }^{1}$ d.benton@aston.ac.uk
}

Keywords: Coherence, laser detection. interferometer

\section{ABSTRACT}

Lasers are an unnatural occurrence, rendered almost impossible in nature due to the laws of thermodynamics. Thus, the presence of laser radiation is always accompanied by an intent for that laser such as sensing, targeting, range finding etc. Detection of laser radiation is therefore important as it may be a precursor to impending action. Laser warning receivers have been around for decades and have been aligned with the type of laser threat. In the last few years new threats have appeared in the form of low-cost diode lasers with dangerously high power levels (several Watts for a few hundred US dollars) and an ever expanding range of wavelengths. Protecting against such threats requires its detection, analysis and classification. In this paper we will discuss the types of technologies that have been used to detect lasers and the properties they can discern. We then focus on the developments in the detection of coherence properties and its ability to detect weak continuous wave (CW) laser sources.

\section{INTRODUCTION}

The original inventors of the laser could hardly have imagined the plethora of laser types and the scale of applications that now utilize lasers. The list of applications includes communication, spectroscopy, drilling, scanning, medical applications, holography, printing and projection to name a few. Lasers are pervasive in the modern world. However, lasers are an unnatural occurrence, rendered almost impossible in nature (in the optical regime) due to the laws of thermodynamics. Thus the presence of laser radiation is always accompanied by an intent for that laser.

In military scenarios that intent could be hostile. The detection and identification of laser radiation is then particularly aligned to the military requirements. In military scenarios lasers are used for targeting, range finding, designation, and missile control [1] as well as dazzle weapons and directed energy weapons. The majority of lasers of interest to the military are pulsed lasers. The temporal resolution and high instantaneous brightness make them well-suited for operating over ranges of many kilometers in applications such as range finders and target markers. Generations of laser warner receivers (LWRs) have been developed to detect the threat posed by these lasers $[2,3]$ and allow irradiated platforms to initiate appropriate countermeasures determined by the perceived threat. In the last few years a more widespread hazard has arisen from the preponderance of continuous wave $(\mathrm{CW})$ visible laser diodes - so called laser pointers. Handheld visible lasers with powers of several Watts are available for a few hundred dollars with an everexpanding range of wavelengths. These lasers have proven to be a menace when directed towards aircraft as they approach a landing, with over 1500 reports of lasers dazzling pilots in the UK and US last year. Conventional LWRs do not perform well at detecting these lasers. CW lasers are therefore a specific challenge in the world of laser detection. This paper outlines a variety of techniques used to detect lasers and then concentrates on work done to utilize coherence characteristics of a laser source as a method of detection.

Technologies for Optical Countermeasures XVI, edited by David H. Titterton, Robert J. Grasso, Mark A. Richardson, Proc. of SPIE Vol. 11161, 111610G · C 2019 SPIE · CCC code: 0277-786X/19/\$21 - doi: 10.1117/12.2532058 


\section{METHODS}

Lasers tend to have three characteristics which serve as the origin of their usefulness as well as discriminating characteristics for detection. These are:

1. Low divergence beams which brings about spatial concentration. Thus imaging systems can observe either a laser spot or a directional dependence.

2. Monochromatic wavelength which leads to a high spectral concentration and can be observed by dispersing the light and looking for narrow peaks in a spectrum. The peaks are usually narrower than the spectral resolution available.

3. Long coherence lengths.

Wang [4] divided laser detection into three categories - coherent recognition, scattering recognition and spectrum recognition. These are categories based on what is observed rather than the discriminating characteristic. Instead here we shall use categories based on discrimination technique - imaging, spectral and coherence. The first two categories are both essentially discriminating based on brightness.

\subsection{Imaging Detection}

Imaging laser detection systems are typically composed of an optical imaging system and an array of detectors such as a CCD [5]. They can detect both CW and pulsed lasers and are able ascertain the direction of origin of the laser. The use of a fisheye lens offers a significant field of view. The BRILLIANT system [1] uses an intensified video camera where the intensifier gate is controlled to optimize the signal-to-noise and to control saturation effects. This requires some understanding of the pulse parameters being used. When CMOS sensors are used the incomplete fill factor can lead to some location errors highlighted by Ying [6,7]. When detecting "brightness" with a large area detector the system can be subject to false alarms from bright sources such as the sun or lightning. Reducing background light by the use of narrow band filters is required which inevitably limits the wavelengths that can be detected. A recent innovative semi-imaging system uses a defocused system and looks for telltale circles of brightness [8]. This can infer source direction and wavelength and is built from low cost components.

\subsection{Spectroscopic detection}

Looking for spectral brightness i.e. well defined narrow peaks in a dispersed spectrum, requires a dispersal system typically a diffraction grating and a detector array. Recent developments have used a sinusoidal grating to diffract incoming laser light into 3 orders $(0, \pm 1)[9,10]$. The direction can be inferred from the position of the central zero order, and the wavelength inferred from the separation of the other orders (see also references in discussion).

\subsection{Coherence Detection}

Coherence is the stability of phase over time and relates to the consistency with which one part of a wave can relate to another part removed in time. This time relationship is translated into a distance using the speed of light. Lasers tend to have long coherence lengths ( $\mathrm{cms}$ to $\mathrm{kms}$ ) in comparison to broadband emissive sources which can have coherence lengths of microns. Thus coherence is both an indicator of laser radiation and a method of discrimination against background sources. Interferometers rely on coherence to work and several interferometers have been applied to the area of laser detection.

The Fabry Perot interferometer was used by Crane [11,12] to detect pulsed and CW lasers. Stepped interferometers were used with the difference signal between the interferometers able to remove common components. The systems were angle scanned causing a modulation of input light which can be used as a signature of coherence [13]. In some cases the fabrication of Fabry Perot interferometers requires specific laser threat wavelengths to be known in advance [14]. Determination of laser wavelength is possible with high accuracy but over a limited spectral bandwidth [15]. 
Michelson interferometers have been used as coherence detectors where long path length differences are able to determine the coherence length of the source [16,17]. When used with an imaging system this is able to remove background illumination [18]. Multiple interferometer paths are able to act as a coherence length bandpass filter [19]. Michelson interferometers are able to provide accurate wavelength measurement as well as coherence length measurements but may be limited in field of view and assessment time.

The Fizeau interferometer has been also been considered [9] [20]. As this has already found application in commercial wavelength meters able to determine wavelength of pulsed lasers to high accuracy, it is a natural extension to inferring its use for laser detection.

Cohen [21] employed a polarization based interferometer using an electro-optic modulator to discriminate against incoherent background light and assess the laser wavelength.

Mach Zehnder interferometers (MZI) have also been used for laser detection by Benton [22], the substance of which forms the remainder of this paper.

In common with many of the above coherence detecting techniques, Benton implemented an asymmetric interferometer to discriminate against incoherent background light. This involves introducing a path length difference between the interferometer paths that exceeds the coherence length of the background light, such that no interference can be observed. For broadband visible light the coherence length is only a few microns and it is relatively easy to implement this path difference (without trying!). One of interferometer arms has a piezo mounted mirror which is sinusoidally modulated by around a quarter of a wavelength. Any source light entering the MZI which has a coherence length longer than the MZI asymmetry will generate a modulated output signal at the frequency of the mirror modulation. Such a modulating signal can only be generated by a long coherence length source and is thus an indicator of a laser of the presence of laser radiation (or an atomic emission line like a sodium lamp). The MZI has 2 output ports which can be used to observe modulations and these signals are $180^{\circ}$ out of phase. A schematic diagram of the system is shown in figure 1.

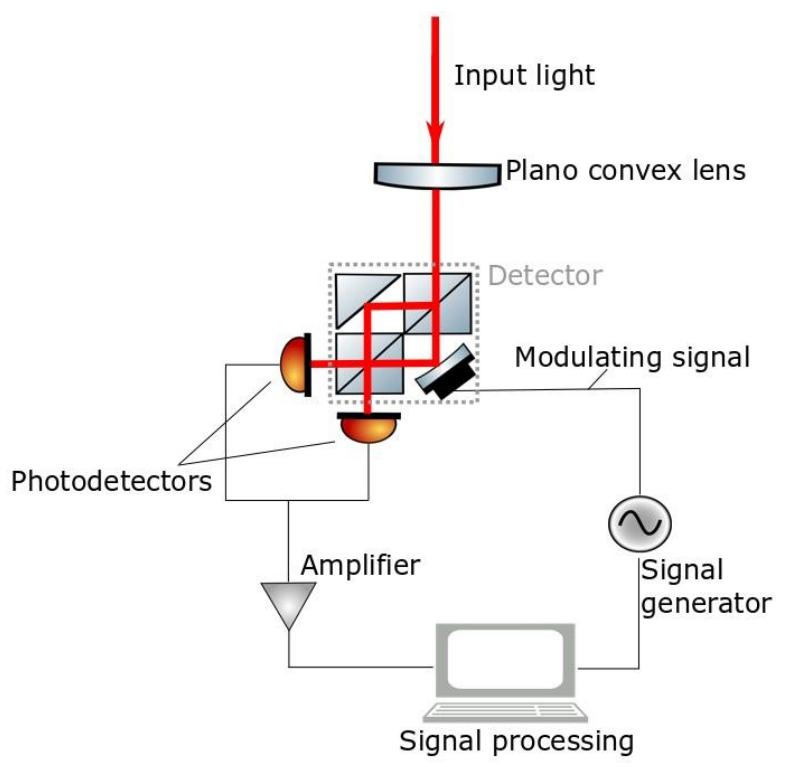

Figure 1. A schematic Diagram of a Mach Zehnder interferometer laser detection system

The driving principle behind this device was to be low cost. Consider the non-coherence detecting systems. The imaging and spectroscopic methods both employ detector arrays, which, in the visible part of the spectrum are low cost, but in the near IR beyond silicon detection band such arrays become a significant cost. The MZI method requires only a single detector that is appropriate for the waveband. In addition, a piezo mirror is a relatively low cost device in comparison with some of the more expensive modulators used in other coherence methods. 


\section{RESULTS}

The output from the detectors at both ports are utilized with a balanced detector. This subtracts the two detector signals, reducing common background and increasing the signal size due to the $180^{\circ}$ phase difference. The amplified signal is sampled with an ADC and the digitized signal is Fourier transformed to produce a frequency spectrum where several spectra can be integrated. The power at the modulation frequency is extracted to ascertain the presence of laser light. This has been used to assess the detection sensitivity using 2 detectors at one or both output ports as shown in figure 2

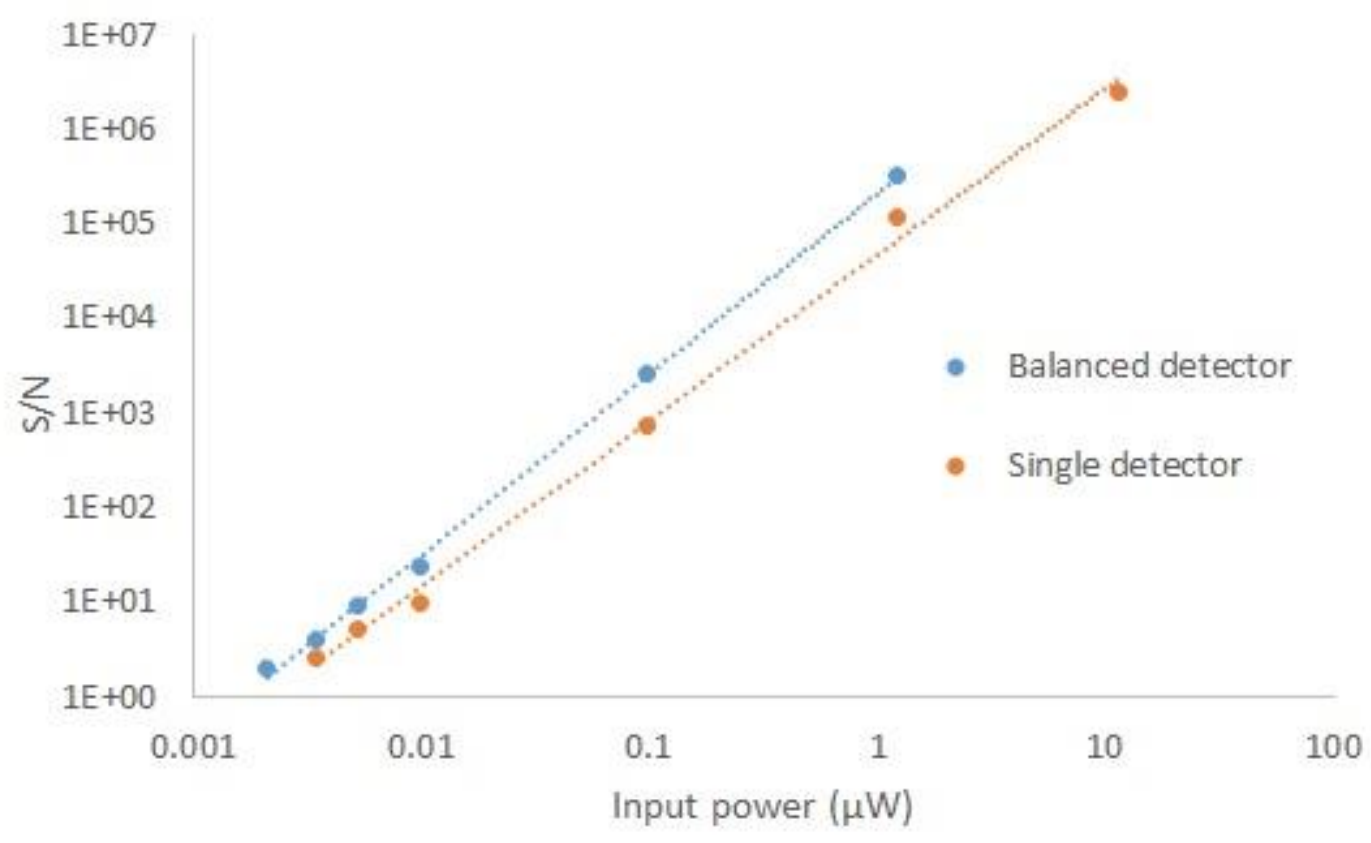

Figure 2. Measured sensitivity of the MZI laser detector for 1 and 2 photodetectors

Extrapolating down to a $\mathrm{S}: \mathrm{N}$ of 1 gives an equivalent sensitivity of $1 \mathrm{nW}$ using two detectors and $2 \mathrm{nW}$ using a single detector. Measurements were made with a diode laser at $635 \mathrm{~nm}$ using large area silicon photodiodes and a modulation frequency of $600 \mathrm{~Hz}$. These measurements were made with controlled dark background. The background light in the detector affects the noise levels generated (shot noise) and ultimately could saturate the device. However one of the benefits of coherence detection is that it can discriminate against background light. This can be seen in figure 3 where a beamsplitter is used to direct a laser and light from an incandescent source into the interferometer. Figure 3 also shows the relative brightness from the other beamsplitter port. The incandescent light is $\mathrm{AC}$ driven and shows $100 \mathrm{~Hz}$ with higher order harmonics. The mirror was modulated at $637 \mathrm{~Hz}$ to discriminate it. As the laser intensity is reduced to below visibility against the background its modulation can still be observed with a power of $70 \mathrm{nW}$.

Modulating with a fixed amplitude means that the phase change experienced by the laser is dependent upon the wavelength. Shorter wavelengths can experience a phase change that exceeds a quarter of a wavelength per cycle. This appears as stronger harmonics of the modulation frequency. It is then possible to determine the wavelength of the incoming light by analysing the ratio of the third harmonic to the fundamental frequency (see Benton [22]). However in some cases this can be unreliable due to large differences in the harmonic powers. This will be discussed in a subsequent publication. 

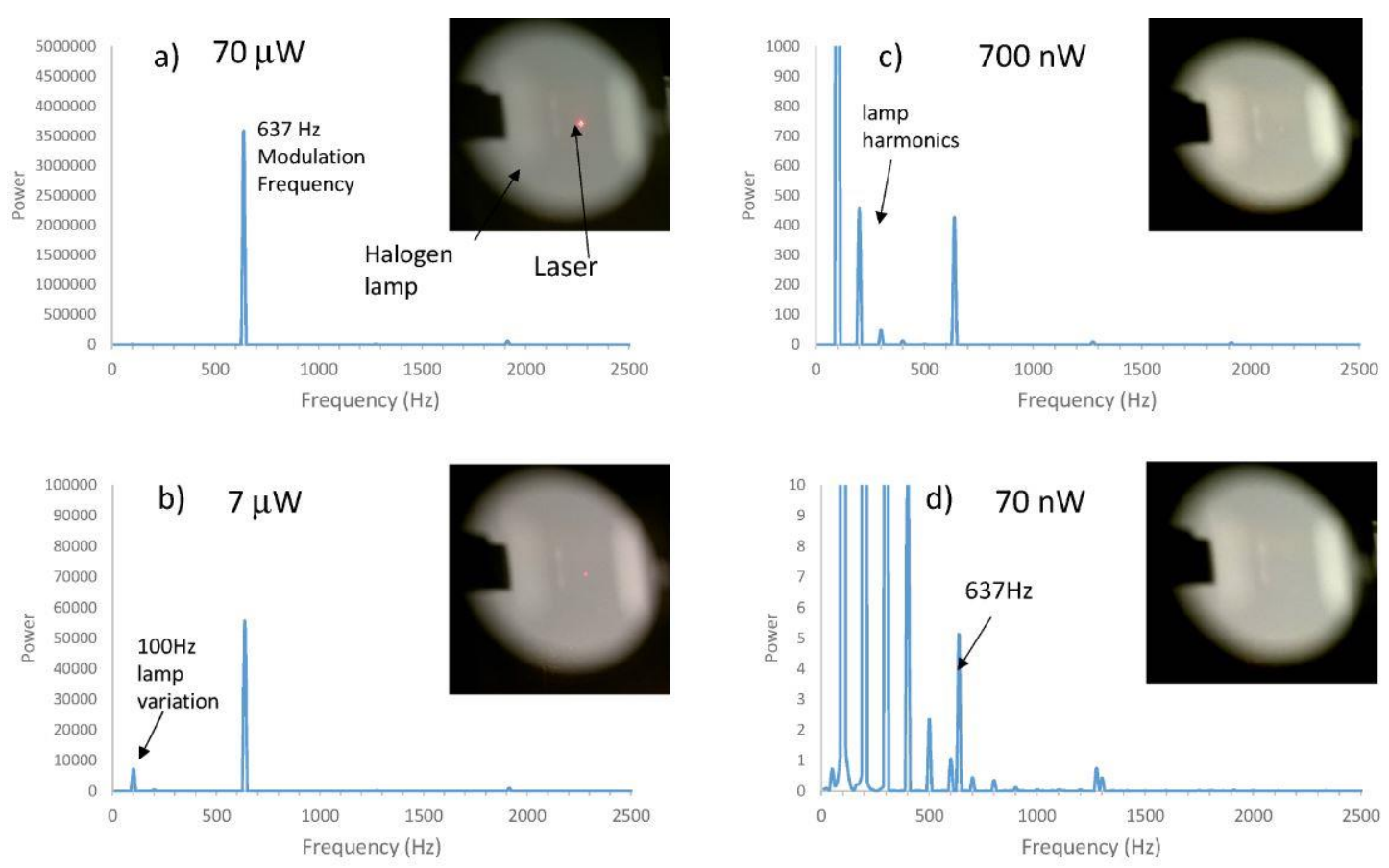

Figure 3. Demonstrating the relative intensities of a halogen lamp and laser, with graphs of the interferometer output frequencies.

\section{DISCUSSION}

Whilst laser warner receiver technology has been around for decades, it matured to detect the prevalent threats of the time. In recent years the accessibility of low cost, high brightness $\mathrm{CW}$ diode laser has produced a widespread, civil requirement for laser detection. It is with this view that the use of laser detection systems based on the detection of coherence will likely find a role. As is often the case the fundamentals of a solution have been well established in the preceding decades but technology has now advanced sufficiently to make those ideas realizable - take for example the ease with which sophisticated digital signal processing is now available on most mobile phones.

Laser detection systems will ideally need to detect both continuous and pulsed lasers, will need to ascertain the source location, determine the laser wavelength and any modulation being applied. For systems based on interferometry, the limited field of view is a significant issue in being able to locate the source and may well result in multiple systems being deployed. A recent development has seen a 360 deg azimuthal field of view coupled with an MZI and is reported elsewhere in these proceedings (by Zandi).

Finally we end the discussion with an extreme extension of where we began. Laser radiation is (as far as we know) not found in nature and therefore its detection is both an indicator of intent and intelligence. Thus it is that the Search for Extraterrestrial Intelligence (SETI) has focused some part of its effort on looking for laser signatures. As with conventional laser detection the approaches taken have been on observing the relative brightness - either in high resolution spectroscopy [23], or in short pulse coincidence detection [24]. Benton [25] has recently shown that coherence detection can be applied to this problem at the level of single photon detection and can offer potential improvements in sensitivity to around 1 photon $/ \mathrm{m}^{2} / \mathrm{s}$ and detection of both CW and pulsed lasers. 


\section{CONCLUSION}

Methods of laser detection based on 3 defining characteristics of laser radiation - spatial brightness, spectroscopic brightness and coherence have been presented, with an emphasis on methods of coherence detection. Interferometric systems have been demonstrated to identify laser radiation based on the property of long coherence length. Such systems are potentially useful in identifying CW diode lasers that are now widely available.

\section{REFERENCES}

[1] Dubois, J., \& Reid, F. "Detecting laser sources on the battlefield". Proc. SPIE, 6796(2007), 67962F (2007). http://doi.org/10.1117/12.779234

[2] Pietrzak, J. "Laser warning receivers". Proc. SPIE, 5229(2), 318-322. (2003)

[3] Dąbrowski, M., Młodzianko, A., Pietrzak, J., Zygmunt, M., \& Niedzielski, R. "Laser warner receiver LWR-H". Proc SPIE, 6598, 65980S-4(2006). http://doi.org/10.1117/12.726581

[4] Wang, L. et al. "Optimum design of wideangle laser detecting system based on fisheye lens and sinusoidal amplitude grating". Optics Communications, 310, 173-178 (2014).

[5] Kumar, S. et al. "Design of a laser-warning system using an array of discrete photodiodes - part I". Journal of battlefield technology 14.1 (2011).

[6] Ying, J. and Zhou, Z. "Study on Image Processing Technology in Imaging Laser Detection System". IEEE (2010).

[7] Ying, J., He, Y., and Zhou Z. "Analysis on Laser Spot Locating Precision affected by CMOS Sensor Fill Factor in Laser Warning System". IEEE (2009).

[8] Tipper, S., Burgess, C., \& Westgate, C. "Novel low-cost camera-based continuous wave laser detection", (May), 12, (2019). https://doi.org/10.1117/12.2518230

[9] McAulay, A. D. "Detecting modulated lasers in the battlefield and determining their direction". Proc. SPIE, 7336, 73361J. (2009). https://doi.org/10.1117/12.819423

[10]Zhang, J., Tian, E., \& Wang, Z. "Research on laser warning receiver based on sinusoidal transmission grating and high speed DSPs". WSEAS Transactions on Circuits and Systems, 5(8), 1366-1371. (2006). https://doi.org/10.1088/1742-6596/48/1/152

[11] Crane Jr, R. "Laser detection by coherence discrimination”. Optical Engineering, 18(2), 182212. (1979).

[12] Crane Jr, R.” The angle-scanned interferometer”. Optical Engineering, 18(2), 182205 (1979)

[13] Siebert, E.T. “Analyzer for coherent radiation”. Pat. 4,309,108. 1982.

[14] Manasson, V.A., Sadovnik, L.S., Parker, J. H. "Laser Warner receiver Based on coherence Discrimination”. IEEE, 46, 45-46. (2001).

[15] Scholl, T. J., Rehse, S. J., Holt, R. A., \& Rosner, S. D. "Broadband precision wavelength meter based on a stepping Fabry-Pérot interferometer". Review of scientific instruments, 75(10), 3318-3326. (2004).

[16] Hickman,D. “An optical sensor based on temporal coherence properties," J. Sci. Instrum. 21, 187-192 (1988).

[17] Coutinho, R. C., French, H. A., Selviah, D. R., Wickramasinghe, D., \& Griffiths, H. D. "Detection of coherent light in an incoherent background [for IRST]”. In 1999 IEEE LEOS Annual Meeting Conference Proceedings. LEOS'99. 12th Annual Meeting. IEEE Lasers and Electro-Optics Society 1999 Annual Meeting (Cat. No. 99CH37009) (Vol. 1, pp. 247-248). IEEE. (1999, November)

[18] Duffey, C. J. and Hickman, D. “An imaging system based on temporal coherence differences,” J. Phys. D: Appl. Phys. 21, S56-S58 (1988).

[19] Sutton, P. “A novel electro-optical remote-sensing technique based on bandpass coherence processing”. J. Phys. 22 (1989).

[20] Russell J.C. “Coherent laser warning system". Pat. 6,151,114. 2000.

[21] Cohen, J. D. "Electrooptic detector of temporally coherent radiation". Applied Optics, 30(7), 874-883. (1991). https://doi.org/10.1364/AO.30.000874

[22] Benton, D.M. "Low-cost detection of lasers". Opt. Eng. 56(11), 114104 (2017).

[23] Tellis, N.K. and Marcy, G.W. "A Search for Laser Emission with Megawatt Thresholds from 5600 FGKM Stars". The Astronomical Journal, 153(6):1-50, (2017).

[24] Hanna, D.S., Ball, J., Covault, C.E, Carson, J.E., Driscoll, D.D., Fortin, P., Gingrich, D.M., Jarvis, A., Kildea, J., Lindner T., et al. "Oseti with stacee: a search for nanosecond optical transients from nearby stars". Astrobiology, 9(4):345-357, (2009).

[25] Benton D.M. "A Proposed Method for a Photon-counting Laser Coherence Detection System to Complement Optical SETI." Publications of the Astronomical Society of the Pacific, 131(1001), 074501. (2019). https://doi.org/10.1088/1538-3873/ab1a46 